\title{
A Weight-based Road Impedance Function Model
}

\author{
Jie Dong, Guojie Shen \\ Department of Control Science and Engineering \\ Dalian University of Technology \\ Dalian, China \\ dongjie@mail.dlut.edu.cn
}

\begin{abstract}
Path planning problem is a popular issues in urban transportation system. Road impedance is one of the key factors in route planning. According to road length and speed, road level, traffic lamp and intersection waiting time that affect drive efficiency. A new weighted road impedance model is proposed. To get the weights of each factor in road planning, factors which influence drive efficiency are tested with different combination. In the end, the impedance model is applied to real world network topology. The simulation results show the validity and accuracy of the model.
\end{abstract}

Keywords-route planning; weighted road impedance model; network topology

\section{INTRODUCTION}

As people travel more and more frequently, they spend more time on transportation. Therefore it becomes a pressing concern to find an optimal path. Traffic path planning problem is usually classified approximately shortest path problem. Network shortest path problem has been put forward as early as 50 years ago and the most classic is the research about shortest distance [1]. But considering physical distance only is not much practical significance in a real urban traffic system. It is assumed that we get a shortest route by algorithm of shortest path. However, poor road conditions or traffic congestion, compared to other path that need much more time to go through. Increased energy consumption on the one hand, on the other hand, waste more time. Therefore, get shortest time [2-4] route is a good choice. In this paper, we consider the optimal path is the shortest time route, that is, the shortest travel time from the beginning to the end.

In order to obtain the shortest path, we need to consider other factors that affect driving efficiency apart from physical distance in the transport system. The paper [5] considers real-time speed, intersection delay and real time road delay. But real-time delay is generally difficult to determine, such as traffic accidents ranging from affecting driving speed, weight will cause traffic jam, causing traffic congestion for several hours. Paper [6] discusses in more detail the modeling problem of the topology of the network of bus lines, and based on a network of bus lines arcs between nodes geographically relevant topological features. As a platform of Mapinfo, obtain the shortest path between any two nodes in the network of bus lines with shortest path algorithm. However, in the calculation of the shortest path, consider only the most important factors of distance. Based on weather, travel, urban transport, security, services, other variables and sub-information of the variable, [7] proposes a hierarchical weighted impedance function model.

Based on analysis above, this paper considers the static road network traffic information comprehensively. For example, the sections of the length, average speed of vehicles in the road, road grade, roads intersections and the number of traffic lights. Then proposed a weighted road resistance function model and discuss the proportion of the various factors in the path planning through experiments, in order to determine the value of the weighted road impedance function. Finally, experiments on real road traffic system, verify the accuracy of the model.

\section{PREPROCESSING}

Urban transport system in the optimal path generally put shortest traveling distance, shortest travel time [8], the lowest level of crowding [9] and best road quality as the goal to calculate the best route between any two nodes of the traffic road network. Must first clear the circumstances in which the optimal calculating the optimal path, and then determine the impedance functions. For example, planning the static shortest distance, the impedance function is the lengths of the road. Planning shortest path impedance, the impedance functions is a variety of factors that affect driving efficiency, to make shortest path planning problem become a more complex problem. To find out proportions of driving factors in path planning, Mapinfo software is used to extract the network topology of the real part of the road in Xian, Shanxi Province, and use dijkstra algorithm validation model results.

\section{A. Influencing factors of travel times}

Considering shortest time as the goal of static route planning, the impedance functions will be integrated considering a variety of factors that affect driving efficiency is as follows:

- $\quad$ Sections of the length and speed: The length and speed of the sections are the most important indicators of travel time in the urban transport system. The shorter link length, the faster sections of speed, the shorter travel time.

- The road grade: The city road grade is divided into fast roads, trunk roads, secondary roads and branch. Road grade, its pavement quality also different, and width of 
the road are also different, making the maximum driving speed of the design differently. The higher the level of road, the better the road quality, the wider the road width, the higher the maximum speed limit, the shorter the travel time of the vehicles on the road.

- Waiting time of the intersection: Vehicles must stop and wait for a red light at the intersection, this will cause intersection waiting time delay. Especially the intersection is of a relatively large, waiting time at the intersection queuing become a higher proportion of the entire trip. So the intersection waiting time is an important factor that cannot be ignored in the city traffic system. A road traffic light more, the intersection wait time is relatively longer.

Therefore, in the path planning, these affect driving efficiency factors embodied in various forms in the network topology, to facilitate the determination of the impedance functions.

\section{B. Extraction of network topology}

In urban transport system, road is usually abstracted into edges in the graph theory [5], to abstract the intersection into the vertex. And the impedance function is the right side in the graph theory. To abstract urban transport system into a network topology, defined as follows:

Define $G=(N, A, C)$ as a Network diagram, where $\quad N=\{1,2, \ldots, n\} \quad, \quad A=\{(i, j) \mid i, j \in N\} \quad$, $C=\left\{c_{i j} \mid(i, j) \in A\right\}$ represent nodes, line segments, and travel time.

Roads are criss-cross in urban transport system. Various streets, fast-track, main roads, secondary roads and slip roads connect to form numerous intersections which as the vertex of road network, various kinds of roads as the side, to obtain the time required in the roads. City road network of Xian is used as experimental data. Because this part of the data have more road intersections, obvious road grade, therefore has a strong representation in the selection of the optimal path.

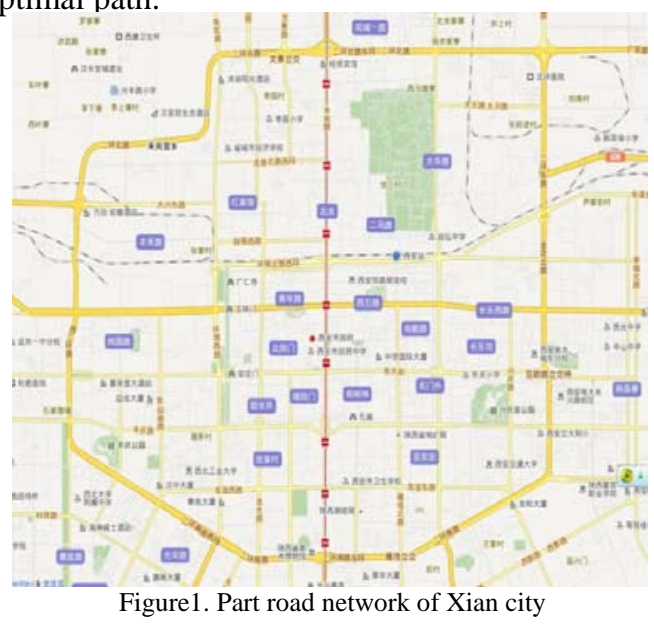

From the figure 1 we can see various loops and roads criss-cross. Outermost coarse yellow line is the fast Ring Road, forming a natural boundary to divide the road network. The smaller and lighter-colored line represents the road of low grade. With west second ring road, south second ring road, east second ring road and north second ring road constitute an approximate rectangular range. The road grade is divided into three according to the actual situation with mapinfo software. Road network is approximated relationship of nodes and edges, as shown in the network topology below, contains 76 nodes and 178 edges.

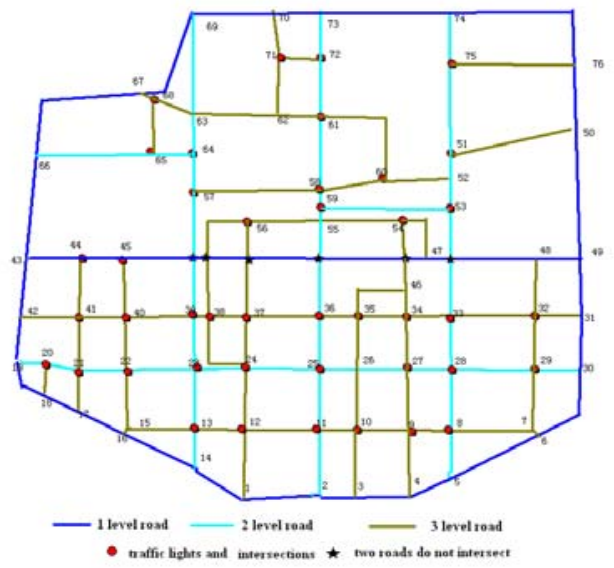

Figure2. Part of road network topology of Xian

\section{Dijkstra algorithm}

After extracting network topology architecture, it is necessary to choose the shortest time path planning algorithm. There are much more path planning algorithm, such as Dijkstra algorithm, neural networks [10], genetic algorithms [11]. Dijkstra's algorithm is known the most improved algorithm theoretically, and also the theoretical basis for the majority of systems used to solve the shortest path problem. It is more suitable for solving the shortest path between two points and has a wide range of applications in the field of transportation. [12] use 10 different states in the USA and 21 different real road network as cases, using different fastest path algorithm to that no matter what data structure are , Dijkstra algorithm has more advantages than the other algorithms in one-to-one, one-to-many paths planning. Dijkstra algorithm obtain shortest path by order of increasing path length. Its scope of application is: searching shortest route from a fixed source to the remaining vertex in the graph. Weight of the topology diagram is nonnegative.

Define shortest path: [13] given a weighted graph $G=(V,\{E\})$, which $V$ is the collection of $\mathrm{n}$ vertices, $\{E\}$ is a collection of $\mathrm{m}$ arcs, $\left\langle V, V^{\prime}>\right.$ is the arcs from vertex $V$ to vertex $V^{\prime}$ in $\{E\} \cdot W<V, V^{\prime}>$ is the nonnegative side of $\left\langle V, V^{\prime}\right\rangle$, assume $\mathrm{a}, \mathrm{b}$ is the vertex of $V, L_{a b}=\left\{v_{0}=a, v_{1}, \ldots v_{n}=b\right\}$ is route from a to $\mathrm{b}$ in $V$, and then the combined weight of route $L_{a b}$ is $W\left(L_{a b}\right)$.Expressed as :

$$
W\left(L_{a b}\right)=\sum_{i=1}^{n-1} W\left(v_{i}, v_{i+1}\right)
$$


The shortest path is the path in a weighted graph that minimize the weights sum of the path, and if the weight is seen as the arc length, that is, from the beginning to the end of the shortest distance of the path. If weight to be seen as a cost, that is, from the beginning to the end least-cost path. And if weights seen as a time, that is the shortest path.

\section{FACTORS DETERMINATION}

Based on driving efficiency factors mentioned in the previous chapter, the shortest path planning is a combination of various factors that affect vehicular efficient, proposed a new weighted impedance function model. Have test on extracted network topology with Dikstra algorithm to determine the impedance function model parameters.

\section{A. Impedance function model}

The length and speed of the road, road grade, waiting time of the intersection, the traffic regulation information, and other factors will affect the travel time. When use shortest time as the goal of route planning between two points, road impedance should consider more than a few factors, in order to simulate a real road traffic conditions. Paper [3] based on the variable weather, travel, urban transport, security, service propose a hierarchical weighted impedance function model. This paper consider the static information of the road network, using the weight division method and variety factors that affect driving efficiency to simulate real urban transportation systems.

Based on the several factors, proposed a weighted impedance function model as follows:

$$
f_{\cos t(\alpha, \beta, \gamma)}=\alpha T_{1}+\beta T_{2}+\gamma T_{3}
$$

Where $f_{\text {cost }}$ represent the travel time between two points, $T_{1}$ is the time decided by the section of length and travel speed. $T_{2}$ is decided by the road grade, $T_{3}$ is decided by the traffic light and intersection time delay. And $\alpha$, $\beta, \gamma$ represent the said weights of the three factors respectively. These three parameters represent the role played by factors in road network of the path planning. And thus conduct experiments and contrast with different combination that use factors which affect driving efficiency.
Therefore determine the right value of the impedance function.

\section{B. Parameters determine of weighted impedance functions}

In order to ensure he right values of impedance function model, it is necessary to analyse the role played by the various factors in the optimal path planning. Considering the length of the road and speed, road grade, intersections and traffic lights extended time without weights, the optimal path is shown as below.

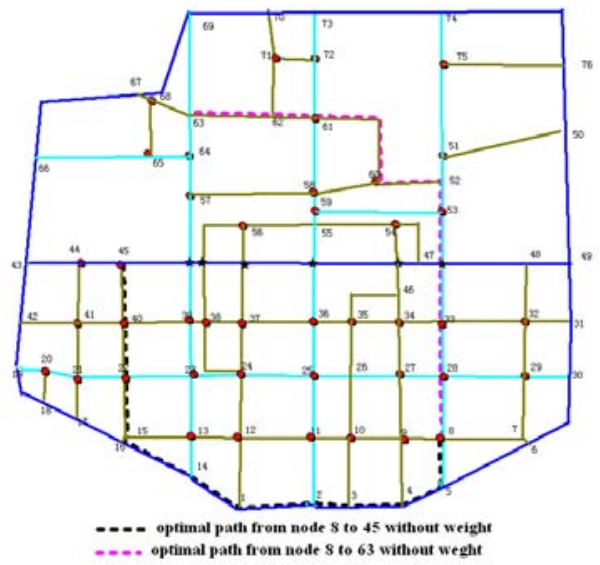

Figure3. Optimal paths from node 8 to 63 and 8 to 45 without weight

It is can be seen from the fig.3, when various factors have the same weight for path planning, the optimal path from the node 8 to node 63 is 8-28-33-53-52-60-61-6263.Meanwhile, the optimal path from the nodes 8-45 is 8-54-3-2-1-14-16-15-22-40-45.Compared with the figure ,two optimal paths are all in the high grade, relatively few traffic lights intersection sections. Now analyse the impact of various factors on the optimal path.

Road length is a basic essential factor regardless of what traffic route planning. D is used to represent road length, $G$ mean road grade and $\mathrm{L}$ represents traffic lights and intersection delay. Therefore, in this paper, DG represents the combination of road length and road grade. Combination of road length and traffic light intersection is expressed in this article by DG. DGL is a combination of the three factors. Therefore get the optimal path under different circumstances as follows:

Paths from 6 to 63 with different combination
\begin{tabular}{|c|c|}
\hline Driving factors & Optimal paths \\
\hline D & $8-9-27-34-46-54-55-59-58-61-62-63$ \\
\hline DG & $8-28-33-53-59-61-62-63$ \\
\hline DL & $8-28-33-53-52-60-61-62-63$ \\
\hline DGL & $8-28-33-53-52-60-61-62-63$ \\
\hline
\end{tabular}

Route contrast from 8 to 63 in different situation

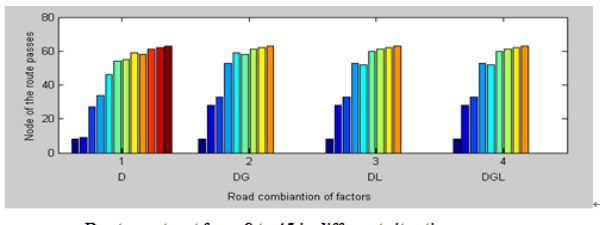

Route contrast from 8 to 45 in different situation

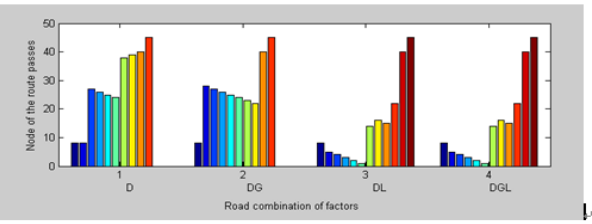

\begin{tabular}{|c|c|}
\multicolumn{2}{|c}{ Paths from 8 to 45 with different combination } \\
\hline Driving factors & Optimal paths \\
\hline D & $8-9-27-34-46-54-55-59-58-61-62-63$ \\
\hline DG & $8-28-33-53-59-61-62-63$ \\
\hline DL & $8-28-33-53-52-60-61-62-63$ \\
\hline DGL & $8-28-33-53-52-60-61-62-63$ \\
\hline
\end{tabular}

Figure4. Comparison under different combinations 
We can see from the comparison of the two sets of tables and graphs, the road length is a fundamental constraint in the path planning, followed by traffic lights and intersections delay. Obviously, road grade played a minor role in real road network path planning. According to the experiment, and the day-to-day experience, the weighted impedance function model is as follows:

$$
f_{\cos t}=0.649 T_{1}+0.263 T_{2}+0.088 T_{3}
$$

\section{ROAD IMPEDANCE FUNCTION MODEL VALIDATION}

Experiment verification with road resistance weights model obtained in the previous section get the result as show in figure 5.

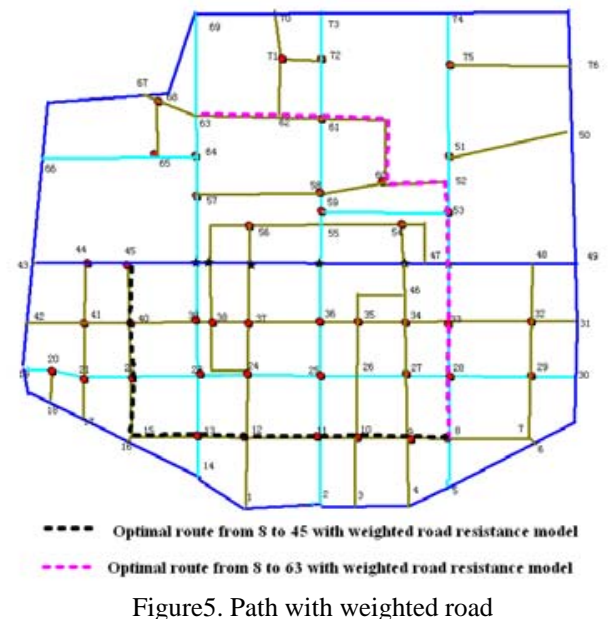

Comparing figure 5 with figure 3 found that the nodes 8-63 get the same optimal path when considering weights and without weight in the impedance functions model. The journey time takes about 828s. However, the optimal path of nodes 8-45 is a low grade, relatively short distance route. As in the cities, due to traffic lights and various traffic factors, the average speed of the vehicle is generally up to less than average speed. Resulting in sometimes vehicles with lower road grade and lower road width have a higher running speed. And the experimental results also verify this feature: TABLE I. TWO DIFFERENT IMPEDANCE MODEL CONTRAST

\begin{tabular}{|l|l|l|l|}
\hline $\begin{array}{l}\text { Endpoint of } \\
\text { path }\end{array}$ & $\begin{array}{l}\text { Run time of } \\
\text { ordinary road } \\
\text { resistance } \\
\text { model }\end{array}$ & $\begin{array}{l}\text { Run time of } \\
\text { weighted } \\
\text { resistance } \\
\text { model }\end{array}$ & $\begin{array}{l}\text { Saving } \\
\text { time } \\
\text { comparison }\end{array}$ \\
\hline $\begin{array}{l}\text { node from 8 } \\
\text { to } 63\end{array}$ & $828 \mathrm{~s}$ & $828 \mathrm{~s}$ & 0 \\
\hline $\begin{array}{l}\text { node from 8 } \\
\text { to } 45\end{array}$ & $729 \mathrm{~s}$ & $687 \mathrm{~s}$ & $6.1 \%$ \\
\hline
\end{tabular}

\section{CONCLUTION}

The paper analyses that if want to go somewhere fast between two nodes in the city traffic system, get the fastest route is a good choice. But what factors influence path planning and how to get the fastest route ?In this paper, based on the analysis of road length and speed, road grade, traffic lights and intersection waiting time delay, proposed a weighted road resistance function model. To determine the impedance function model parameters, have tests with driving efficiency factor in a combined way. Therefore obtain the proportion of the various factors in the path planning. Finally, in the real road network topology of Xian, Shanxi province, experiments simulations verify the accuracy of the model.

\section{ACKNOWLEDGMENT}

The work in this paper was supported by Natural National Science Foundation of China (Number 61004040).

\section{REFERENCES}

[1] E. W. Dijkstra. A note on two problems in connexion with graphs, A note on two problems in connexion with graphs, 1959, pp. 267-271.

[2] F. Lu, D.M. Xu, W.H. Cui, "Time shortest path algorithm for restricted searching area in transportation networks," Journal of image and graphics, Oct.1999, pp. 849-853.

[3] G. Y. Wang, F.Q. Pan, L.X. Zhang, X.D. Zhou, "Optimal path algorithm of road network with traffic restriction," Journal of traffic and transportation engineering, May 2005, pp. 92-95.

[4] J. Halpern, "The shortest route with time dependent length of edges and limited delay possibilities in nodes," Mathematical methods of operations research, vol.21, 1997, pp. 117-124.

[5] G.M. Zhang, "Study on optimal path algorithm for GPS/GIS vehicle navigation system,” Journal of sichuan normal university, vol.28, 2008, pp. 497-500.

[6] D.F. Ren, A.G. Xu, "Road topology and the optimal route realization based on MapInfo", Engineering of surveying and mapping, vol.20, 2011, pp. 53-56.

[7] S.N. Abolghasem, V. Masood, K. Kyehyun, J.J. Jason, "Real world representation of a road network for route planning in GIS," Expert systems with applications, vol. 38 ,2011, pp. 11999-12008.

[8] J. Husdal, Network analysis - Network versus vector - A comparison study, UK, University of leicester, 2000.

[9] J.D. Leonard, M. Oliveira, "Towards an areawide service measure," In Proceedings of the Fourth International Symposium on Highway Capacity, Transportation research board, 2000, pp. 17-25.

[10] L. Chen, W. He, L.Q. Han, "Study on urban transport optimal path algorithm," Transactions on intelligent systems, vol.7, 2012, pp. 1-7.

[11] Q. Li, W. Zhang, Y.X. Yin, Z. L. Wang, "An improved genetic algorithm for optimal path planning,” Information and control, vol.35, 2006, pp. 444-447.

[12] F.B. Zhan, C.E. Noon, "Shortest path algorithms: an evaluation using real road networks,” Transportation science, vol.32, 1996, pp. 65-73.

[13] X.G. Zhang, Q. Wang, N. Wang, "A study on road network model in digital maps and the automatic generation algorithm of its database," Journal of image and graphics, vol.6, 2001, pp. 481-485. 